\title{
Meloxicam associado ou não ao tramadol no controle da dor após ovário-histerectomia videoassistida em cadelas
}

[Meloxican with or without tramadol for control of pain undergoing laparoscopic-assisted ovariohysterectomy in bitches]

\author{
M.T. Oliveira ${ }^{1}$, R.T. França ${ }^{1}$, J.P.S. Feranti ${ }^{1}$, A.S. Coutinho Júnior ${ }^{1}$, A.V. Soares ${ }^{1}$, \\ F.R. B. Santos ${ }^{2}$, L.F.D. Corrêa ${ }^{1}$, H.F. Hartmann ${ }^{1}$, S.T.L. Pinto Filho ${ }^{1}$, \\ R.O. Chaves ${ }^{1}$, V.H. Pohl ${ }^{1}$, M.V. Brun ${ }^{1,3^{*}}$ \\ ${ }^{1}$ Universidade Federal de Santa Maria \\ ${ }^{2}$ Universidade Federal do Paraná \\ ${ }^{3}$ Bolsista de produtividade do $\mathrm{CNPq}$
}

\begin{abstract}
RESUMO
O objetivo deste estudo foi avaliar a eficácia do meloxicam associado ou não ao tramadol, no controle da dor após ovário-histerectomia $(\mathrm{OVH})$ laparoscópica com dois portais. Foram selecionadas 14 cadelas hígidas. Os animais foram separados de forma aleatória, em dois grupos. O grupo M (GM) recebeu meloxicam (0,2 $\mathrm{mg} \mathrm{kg}^{-1}$, s.i.d.), enquanto os animais do grupo MT (GMT) receberam a associação de meloxicam (0,2 $\mathrm{mg} \mathrm{kg}^{-1}$, s.i.d.) e tramadol $\left(4 \mathrm{mg} \mathrm{kg}^{-1}\right.$, t.i.d.), ambos durante dois dias de pós-operatório. Para avaliação da dor pós-cirúrgica, foram utilizadas as escalas de Melbourne e escala visual analógica (EVA), além de mensurações de glicemia e cortisol sérico. Não houve diferença ao se avaliarem os grupos GM e GMT pela escala de Melbourne nem pela EVA. As mensurações de cortisol não atingiram valores superiores aos de referência para a espécie, enquanto os valores de glicemia não apresentaram variação significativa ao longo do tempo de avaliação nem entre grupos. Com os resultados deste estudo, foi possível concluir que a utilização de meloxicam associado ou não ao tramadol, nas doses e posologias propostas, é eficaz para controlar a dor pós-operatória de cadelas submetidas à OVH laparoscópica com dois portais.
\end{abstract}

Palavras-chave: cão, analgesia, AINES, videocirurgia

\begin{abstract}
The aim of this study was to evaluate the efficacy of meloxicam with or without tramadol for pain control after laparoscopic-assisted ovariohysterectomy $(\mathrm{OVH})$ by two-portal access. Were selected 14 healthy dogs to perform video-assisted OVHs. The animals were divided randomly into two groups (GM and GMT). The GM group received meloxicam $\left(0.2 \mathrm{mg} \mathrm{kg}^{-1}\right.$, s.i.d), whereas the GMT group received the combination of meloxicam $\left(0.2 \mathrm{mg} \mathrm{kg}^{-1}\right.$, s.i.d) and tramadol $\left(4 \mathrm{mg} \mathrm{kg}^{-1}\right.$, tid), both for two days after surgery. To evaluate the post-surgical pain Melbourne and EVA scales were used, and blood glucose and serum cortisol were measured. There was no statistical difference when evaluating GM and GMT groups and the Melbourne scale or the visual analogue scale VAS. Cortisol measurements did not reach values higher than the reference for the species, while blood glucose levels did not present significant statistical variation throughout the evaluation time or between groups. With these results, we concluded that the use of meloxicam with or without the tramadol at the doses and dosage schedules proposed, is effective to control postoperative pain in bitches that had undergone video-assisted OVH with two-portal access.
\end{abstract}

Keywords: dog, analgesia, AINES, videosurgery

Recebido em 21 de março de 2015

Aceito em 7 de agosto de 2015

*Autor para correspondência (corresponding author)

E-mail: mauriciovelosobrun@hotmail.com 


\section{INTRODUÇÃO}

A ausência ou deficiência no tratamento da dor pode desencadear hiperalgesia, sofrimento permanente e, dependendo da gravidade, levar à morte (Penning, 1996).

Muitos estudos visam ao desenvolvimento de novas técnicas operatórias, bem como de medicamentos que diminuam o sofrimento dos animais no período pós-cirúrgico (Otero, 2005; Luz, 2009). Nesse contexto, a videocirurgia, por suas características de menor lesão tecidual, menor resposta inflamatória e consequente redução da dor no pós-operatório (Freeman et al., 2010), vem sendo amplamente utilizada.

A combinação de opioides e anti-inflamatórios não esteroidais (AINES) pode ser empregada com sucesso no pós-operatório de ováriohisterectomia (OVH) convencional (Shih et al., 2008). No entanto, apesar de a OVH laparoscópica em pequenos animais ser realizada de maneira rotineira em algumas instituições de ensino, a literatura é carente de protocolos específicos de analgesia empregados para abordagens laparoscópicas.

Os fármacos agonistas opioides são amplamente utilizados no tratamento de dor pós-operatória (Pereira e Marques, 2009; Almeida et al., 2013; Teixeira et al., 2013) pelo seu reconhecido potencial analgésico. A eficácia dos opioides pode variar segundo a espécie, a dosagem utilizada, a característica e a duração da intensidade do estímulo, por bloquear as transmissões periférica e central da via nociceptiva aferente, e, por isso, eles se tornam bastante eficientes no tratamento da dor inflamatória aguda (Ribeiro et al., 2002).

McCormack (1994) relata que os antiinflamatórios não esteroidais (AINES) possuem marcado efeito analgésico e não apresentam os efeitos colaterais dos opioides, como depressão respiratória, redução da motilidade gastrintestinal e os efeitos psicomotores. Porém, Pereira e Marques (2009) demonstraram que o uso de meloxicam foi ineficiente no controle da dor pósOVH convencional, quando comparado à analgesia promovida pela administração de xilazina ou morfina pela via epidural.

O objetivo deste estudo foi avaliar a eficácia do meloxicam associado ou não ao tramadol no controle da dor pós-OVH videoassistida com dois portais.

\section{MATERIAL E MÉTODOS}

O experimento foi realizado após a aprovação da Comissão de Ética no Uso de Animais em Ensino e Pesquisa (CEUA) da Universidade Federal de Santa Maria (UFSM), conforme o parecer 081/2012.

Os cães foram obtidos a partir da rotina do Hospital Veterinário Universitário da UFSM e de proprietários que tinham interesse em realizar a castração eletiva de seus animais, mediante autorização por escrito e completa explanação do procedimento a ser realizado.

Foram selecionadas 14 cadelas hígidas, adultas, pesando em média $8,47 \pm 4,23 \mathrm{~kg}$. Os animais foram separados de forma aleatória em dois grupos. O grupo $\mathrm{M}(\mathrm{GM})$ recebeu meloxicam (0,2mg $\mathrm{kg}^{-1}$, s.i.d.), durante dois dias de pósoperatório, enquanto os animais do grupo MT (GMT) receberam a associação de meloxicam (0,2mg kg $\mathrm{kg}^{-1}$, s.i.d.) e tramadol (4mg kg-1, t.i.d.), durante dois dias. Em ambos os grupos foi administrada a primeira dose das medicações, por via intravenosa, imediatamente após o término da cirurgia, e as subsequentes foram administradas por via subcutânea.

As pacientes foram submetidas à avaliação clínica, quando foram computados valores de frequência cardíaca (FC) e frequência respiratória $(f)$, aferidos com auxílio de estetoscópio. Também foram computados valores de pressão arterial sistólica (PAS), por meio de Doppler ultrassônico, com utilização de manguitos de diâmetro adequado para cada animal (30-50\% do diâmetro do braço), além de aferição de temperatura retal. A avaliação hematológica pré-cirúrgica incluiu hemograma, contagem de plaquetas e testes bioquímicos séricos para mensuração de creatinina, albumina e alanina aminotransferase. Também foram mensurados glicose sérica e cortisol plasmático no pré-operatório, mediante coleta de amostra sanguínea no momento em que o animal foi manipulado para a administração da prémedicação, cujos valores constituíram os parâmetros basais.

O experimento foi realizado em duas etapas, sendo inicialmente realizadas as OVHs e, logo 
após, a avaliação da dor e do desconforto pósoperatórios. Para tanto, os animais passaram por período de 24 horas de adaptação ao ambiente experimental e ao contato com os observadores antes da realização do procedimento cirúrgico.

Foi instituído jejum pré-operatório sólido de 12 horas e hídrico de seis horas. Os pacientes foram tranquilizados com acepromazina $\left(0,05 \mathrm{mg} \cdot \mathrm{kg}^{-1}\right.$, i.m.). Decorridos 15 minutos, realizou-se a tricotomia, seguida por encaminhamento ao bloco cirúrgico. A indução anestésica foi obtida pela aplicação de propofol $\left(4 \mathrm{mg} \cdot \mathrm{kg}^{-1}\right.$, i.v. ou dose suficiente para permitir a intubação orotraqueal), e a manutenção foi feita com isoflurano em oxigênio $100 \%$, em concentração adequada para manter os animais em plano anestésico moderado, com ventilação assistida e vaporizador calibrado. Associou-se ainda a infusão intravenosa contínua de fentanila $\left(20 \mu \mathrm{g} \cdot \mathrm{kg}^{-1} \cdot \mathrm{h}\right)$, por meio de bomba de infusão peristáltica, precedida de uma dose bolus de $2,5 \mu \mathrm{g} \cdot \mathrm{kg}^{-1}$ (i.v.). A administração de ampicilina (20mg. $\mathrm{kg}^{-1}$, i.v.) foi realizada 30 minutos antes do início do procedimento cirúrgico. Os animais receberam ringer lactato de sódio $\left(10 \mathrm{~mL} \cdot \mathrm{kg}^{-1} \cdot \mathrm{h}^{-1}\right.$, i.v.) até o momento da extubação. Todos os pacientes foram operados pelo mesmo cirurgião, proficiente na técnica proposta.

Para a realização das OVHs videoassistidas, promoveu-se pneumoperitônio a $12 \mathrm{mmHg}$ com $\mathrm{CO}_{2}$ medicinal, ao fluxo de $1,5 \mathrm{~L} \cdot \mathrm{min}^{-1}$. Foi adotada a técnica de dois portais de acesso de $10 \mathrm{~mm}$, e, para hemostasia dos mesovários, utilizou-se diatermia bipolar. Os ovários, os cornos uterinos e o corpo do útero foram pinçados e exteriorizados por meio do segundo portal de acesso para posterior hemostasia uterina, obtida pela técnica das três pinças. A higienização das feridas cirúrgicas foi realizada com $\mathrm{NaCl} 0,9 \%$, diariamente, durante sete dias, para posterior remoção das suturas.

A avaliação da analgesia pós-cirúrgica foi realizada por dois observadores proficientes na avaliação de dor, cegos ao protocolo analgésico utilizado e à técnica cirúrgica empregada, já que as feridas de acesso e o abdômen foram cobertos por bandagens.

$\mathrm{Na}$ avaliação da analgesia, foram utilizadas as escalas visual analógica (EVA) e da Universidade de Melbourne, iniciando aos 60 minutos após o final da cirurgia; em intervalos de uma hora, das primeiras oito horas (T1 a T8) às 12 horas de pós-operatório (T12); e, posteriormente, a cada 12 horas, até completar 48 horas de avaliação pós-cirúrgica (T24, T36, T48).

Para avaliação empregando a EVA, foi utilizada uma ficha de papel retilínea com $100 \mathrm{~mm}$ de comprimento, sendo uma das extremidades considerada como ausência de dor e a outra como a pior dor possível. Foram observados tanto o comportamento do animal na gaiola quanto o comportamento dele solto na sala de avaliação, levando em consideração a atividade, o status mental, a postura e a vocalização. Em seguida, foi assinalado, na linha, pelos observadores, individualmente e sem que o outro avaliador soubesse, o valor correspondente ao grau de dor, bem como foi realizada a média de pontos entre eles. Quando imputados valores acima de $50 \mathrm{~mm}$, realizou-se analgesia resgate com tramadol (4mg kg-1, i.m.).

$\mathrm{Na}$ avaliação por meio da escala de Melbourne, além da observação do comportamento do animal e da palpação da incisão cirúrgica, foram mensurados os valores de FC, $f$, PAS e temperatura retal, bem como a presença de salivação e dilatação pupilar, pontuadas conforme as categorias avaliadas na escala previamente descrita por Firth e Haldane (1999). Tal escala enquadra valores entre zero, como ausência de dor, e 27, como pior dor possível. A avaliação anterior ao procedimento cirúrgico serviu como valor basal para posterior comparação. Propôs-se realizar analgesia resgate com tramadol (4mg kg-1, i.m.) caso algum animal atingisse pontuação acima de 13, levando em consideração essa escala (Firth e Haldane, 1999).

A administração de resgate analgésico foi instituída quando os animais apresentaram pontuação compatível com dor moderada em pelo menos uma das escalas. Caso os animais necessitassem de analgesia suplementar, seriam removidos das avaliações subsequentes.

Paralelamente, foi mensurada a glicose nos seguintes tempos: T0 (após a indução anestésica), T1, T6, T12 e T24 (horas de pósoperatório); a dosagem de cortisol plasmático foi realizada no T6, T12 e T24 (horas de pósoperatório), em cinco animais de cada grupo. 
Obteve-se o material por meio de catéter heparinizado, firmemente fixado na veia jugular direita, posicionado após a estabilização do plano anestésico. Ambos tiveram como valor basal a amostra coletada no pré-operatório.

Todos os animais do experimento ficaram internados e sob monitoramento durante as primeiras 48 horas pós-cirúrgicas; posteriormente, receberam alta hospitalar.

Para análise dos dados deste estudo, foi realizado o teste de Shapiro-Wilk ao nível de $5 \%$ de significância, e as variáveis não apresentaram resíduos com distribuição normal. Portanto, decidiu-se realizar a análise de variância pelo teste não paramétrico de Kruskal-Wallis para comparação múltipla e, posteriormente, o teste de Dunn para comparar os tratamentos dois a dois, considerando o nível de significância de $5 \%$.

\section{RESULTADOS E DISCUSSÃO}

Os animais de ambos os grupos não apresentaram complicações no transoperatório nem houve necessidade da administração de analgesia suplementar nesse período. O tempo cirúrgico foi de $36,07 \pm 9,03$ minutos.

A média de escores do GM e do GMT não atingiu pontuação equivalente a dor moderada pela escala de Melbourne, nem pela EVA. Porém, um animal do GMT necessitou de resgate analgésico às seis horas de pós-operatório. Esse animal seguiu sendo avaliado, apesar de não participar da análise estatística a partir desse momento. Na sequência, não apresentou novos episódios de resgate. A necessidade de analgesia suplementar nesse caso foi atribuída a fatores individuais, como menor limiar a dor, já que nenhum dos animais dos dois grupos necessitou desse complemento e é reconhecido que tanto dores do tipo somática como visceral respondem bem a anti-inflamatórios não esteroidais e opioides (Lester e Gaynor, 2000).

Ao se avaliarem os animais do GM e do GMT pela escala de Melbourne (Tab. 1) e pela EVA (Tab. 2), não foi evidenciada diferença estatística entre eles, o que demonstra a eficácia de ambos os protocolos analgésicos utilizados.

Tabela 1. Médias de escores e desvios-padrão obtidos mediante o uso da escala de Melbourne na avaliação de dor após OVH videoassistida com dois portais em cadelas que receberam meloxicam (GM) ou meloxicam associado a tramadol (GMT), durante 48 horas de pós-operatório

\begin{tabular}{lccc}
\hline Tempo $(\mathrm{h})$ & $\mathrm{GM}$ & $\mathrm{GMT}$ & $\mathrm{P}$-valor*** \\
\hline Basal & $0,00 \pm 0,00$ & $0,00 \mathrm{~b} * \pm 0,00$ & -- \\
1 & $2,86 \pm 1,31$ & $2,64 \mathrm{a} \pm 1,86$ & $\mathrm{P}=0,80$ \\
2 & $3,07 \pm 1,37$ & $1,93 \mathrm{a} \pm 1,43$ & $\mathrm{P}=0,17$ \\
3 & $3,00 \pm 0,65$ & $2,07 \mathrm{a} \pm 1,43$ & $\mathrm{P}=0,19$ \\
4 & $2,07 \pm 0,93$ & $1,57 \mathrm{ab} \pm 1,79$ & $\mathrm{P}=0,11$ \\
5 & $2,21 \pm 1,22$ & $2,50 \mathrm{a} \pm 3,30$ & $\mathrm{P}=0,19$ \\
6 & $2,29 \pm 1,25$ & $1,79 \mathrm{ab} \pm 3,21$ & $\mathrm{P}=0,11$ \\
7 & $1,93 \pm 1,48$ & $0,58 \mathrm{ab} \pm 0,49$ & $\mathrm{P}=0,11$ \\
8 & $2,14 \pm 1,37$ & $0,67 \mathrm{ab} \pm 0,75$ & $\mathrm{P}=0,06$ \\
12 & $1,43 \pm 1,27$ & $0,42 \mathrm{ab} \pm 0,49$ & $\mathrm{P}=0,06$ \\
24 & $1,50 \pm 1,04$ & $0,42 \mathrm{ab} \pm 0,80$ & $\mathrm{P}=0,09$ \\
36 & $1,29 \pm 0,99$ & $0,58 \mathrm{ab} \pm 0,80$ & $\mathrm{P}=0,21$ \\
48 & $1,57 \pm 1,27$ & $0,50 \mathrm{ab} \pm 0,84$ & $\mathrm{P}=0,11$ \\
$\mathrm{P}$-valor** & $\mathrm{P}=0,07$ & $\mathrm{P}=0,00$ &
\end{tabular}

*Médias seguidas de letras iguais não diferem entre si pelo teste de Dunn ao nível de 5\% de significância.

**Teste de Kruskal-Wallis ao nível de 5\% de significância.

***Teste de Mann-Whitney. 
Os anti-inflamatórios não esteroidais, como o meloxicam, são seguros para mucosa gastroduodenal de cães saudáveis (Costa et al., 2007) e podem ser vantajosos para controlar a dor pós-operatória de $\mathrm{OVH}$ videoassistida, já que não foi evidenciada diferença entre os animais que receberam esse fármaco associado ao tramadol, demonstrando sua eficácia no controle da dor após esse tipo de procedimento de mínima invasão. Outras vantagens da utilização do meloxicam de maneira isolada referem-se à frequência de administração (por ser aplicado a cada 24 horas), ao baixo custo do tratamento, além da ausência dos efeitos colaterais atribuídos aos opioides.

Pereira e Marques (2009) demonstraram que o meloxicam não foi eficaz para o controle de dor pós-OVH convencional. Acredita-se que resultados diferentes foram encontrados no presente estudo pelas características de menor lesão tecidual e de menor estímulo álgico atribuídos aos procedimentos videocirúrgicos quando comparados aos convencionais.

Alguns autores reportam que a intensidade da dor aguda pós-operatória torna-se maior das seis às 24 horas após o procedimento (Mathews, 1996; Hansen, 1997), vindo de encontro aos dados obtidos neste trabalho, pois, ao se avaliarem os grupos pela escala de Melbourne, no GM, ao longo do tempo, não foram computados escores que diferiram do basal, o que sugere que os animais não apresentavam dor alguma. Porém, no GMT observou-se que os maiores escores foram computados até a quinta hora de pós-operatório, diferindo do basal, o que sugere reduzido estímulo álgico promovido pela técnica cirúrgica empregada e/ou eficácia dos protocolos de analgesia utilizados.

Tabela 2. Médias de escores e desvios-padrão obtidos mediante o uso da EVA na avaliação de dor após $\mathrm{OVH}$ videoassistida com dois portais em cadelas que receberam meloxicam (GM) ou meloxicam associado a tramadol (GMT), durante 48 horas de pós-operatório

\begin{tabular}{lccc}
\hline Tempo $(\mathrm{h})$ & $\mathrm{GM}$ & $\mathrm{GMT}$ & $\mathrm{P}-\mathrm{VALOR} * * *$ \\
\hline Basal & $0,00 \mathrm{~b}^{*} \pm 0,00$ & $0,00 \mathrm{~b} \pm 0,00$ & -- \\
1 & $13,21 \mathrm{ab} \pm 2,55$ & $17,14 \mathrm{ab} \pm 6,24$ & $\mathrm{P}=0,48$ \\
2 & $16,43 \mathrm{ab} \pm 2,60$ & $18,29 \mathrm{a} \pm 3,86$ & $\mathrm{P}=0,48$ \\
3 & $18,29 \mathrm{ab} \pm 4,20$ & $19,00 \mathrm{a} \pm 4,42$ & $\mathrm{P}=0,85$ \\
4 & $20,43 \mathrm{a} \pm 4,27$ & $18,71 \mathrm{a} \pm 5,82$ & $\mathrm{P}=0,48$ \\
5 & $20,43 \mathrm{a} \pm 5,42$ & $19,64 \mathrm{a} \pm 8,01$ & $\mathrm{P}=0,61$ \\
6 & $19,79 \mathrm{a} \pm 4,74$ & $22,00 \mathrm{a} \pm 14,18$ & $\mathrm{P}=0,31$ \\
7 & $18,79 \mathrm{a} \pm 5,51$ & $15,50 \mathrm{ab} \pm 3,26$ & $\mathrm{P}=0,17$ \\
8 & $18,43 \mathrm{ab} \pm 8,27$ & $14,33 \mathrm{ab} \pm 2,94$ & $\mathrm{P}=0,39$ \\
12 & $14,50 \mathrm{a} \pm \pm 6,14$ & $13,58 \mathrm{ab} \pm 4,05$ & $\mathrm{P}=0,72$ \\
24 & $7,71 \mathrm{~b} \pm 2,32$ & $8,17 \mathrm{ab} \pm 2,84$ & $\mathrm{P}=0,99$ \\
36 & $6,29 \mathrm{~b} \pm 2,25$ & $7,50 \mathrm{ab} \pm 4,29$ & $\mathrm{P}=0,77$ \\
48 & $5,29 \mathrm{~b} \pm 2,78$ & $4,83 \mathrm{~b} \pm 3,34$ & $\mathrm{P}=0,43$ \\
$\mathrm{P}$-valor** & $\mathrm{P}=0,00$ & $\mathrm{P}=0,00$ & \\
\hline
\end{tabular}

*Médias seguidas de letras iguais não diferem entre si pelo teste de Dunn ao nível de $5 \%$ de significância.

**Teste de Kruskal-Wallis ao nível de 5\% de significância.

***Teste de Mann-Whitney.

Ao se avaliarem os animais pela EVA, os pacientes do GM atingiram pontuação que diferiu do basal das quatro às sete horas de pósoperatório, enquanto os animais do GMT apresentaram os maiores escores das duas às seis horas de pós-operatório. Esse retorno mais precoce aos valores basais pode estar relacionado à característica de menor dor pós-operatória atribuída aos procedimentos videocirúrgicos, quando comparados a técnicas convencionais (Basso et al., 2014).
A dor pós-operatória e o trauma cirúrgico podem provocar as ativações neuroendócrina e metabólica, o que leva ao hipermetabolismo, à aceleração de reações bioquímicas e ao catabolismo orgânico (Bonica, 1992). O cortisol é um parâmetro que pode ser utilizado para avaliação da resposta neuroendócrina ao estresse cirúrgico, podendo auxiliar na indicação da presença da dor (Day et al., 1995; Smith et al., 
1996). No entanto, os procedimentos cirúrgicos minimamente invasivos, como as videocirurgias, podem evitar ou diminuir as alterações fisiológicas e endócrino-metabólicas (Bonica, 1992), fato constatado em ambos os grupos (GM e GMT), já que, em nenhum momento de avaliação, foram computados valores acima dos parâmetros de referência para a espécie (Tab. 3).
Os maiores valores de cortisol foram observados nas coletas basais, obtidas após 24 horas de adaptação dos animais ao local onde iriam permanecer durante o estudo, sugerindo que o tempo de adaptação pudesse ser maior, na tentativa de reduzir as influências atribuídas à troca de ambiente.

Tabela 3. Dosagens séricas médias de cortisol em cadelas submetidas à OVH laparoscópica com dois portais, que receberam meloxicam (GM) ou meloxicam associado a tramadol (GMT), para o controle da dor pós-operatória observada durante 24 horas de pós-operatório

\begin{tabular}{lccc}
\hline Tempo $(\mathrm{h})$ & $\mathrm{GM}$ & GMT & $\mathrm{P}$-valor** \\
\hline Basal & $5,40 \mathrm{a}^{*} \pm 2,41$ & $5,00 \pm 2,24$ & $\mathrm{P}=0,75$ \\
6 & $1,40 \mathrm{~b} \pm 0,89$ & $4,40 \pm 1,82$ & $\mathrm{P}=0,02$ \\
12 & $3,00 \mathrm{ab} \pm 2,35$ & $2,50 \pm 0,58$ & $\mathrm{P}=0,79$ \\
24 & $1,80 \mathrm{ab} \pm 1,30$ & $3,50 \pm 1,73$ & $\mathrm{P}=0,10$ \\
P-valor* & $\mathrm{P}=0,03$ & $\mathrm{P}=0,26$ & \\
\hline
\end{tabular}

*Teste de Kruskal-Wallis ao nível de 5\% de significância.

**Teste de Mann-Whitney ao nível de 5\% de significância.

Apesar de a elevação do cortisol determinar ainda aumento da gliconeogênese hepática e, consequentemente, dos níveis de glicose sanguínea (Breazile, 1987; Lamont e Tranquilli, 2000) - condição observada por outros autores (Stedile et al., 2009) -, neste estudo, os níveis de glicose não diferiram entre os grupos avaliados e se comportaram de maneira semelhante ao longo dos tempos de avaliação (Tab. 4). Esse resultado pode estar associado ao fato de o cortisol ter se apresentado dentro dos parâmetros fisiológicos para a espécie, que variam de 0,5 a $6 \mu \mathrm{g} \cdot \mathrm{dL}^{-1}$ (Feldman, 1996), não sendo suficiente para desenvolver um quadro de hiperglicemia.

Tabela 4. Dosagens séricas médias de glicose em cadelas submetidas à OVH videoassistida com dois portais, que receberam meloxicam (GM) ou meloxicam associado a tramadol (GMT), para o controle da dor pós-operatória observada durante 24 horas de pós-operatório

\begin{tabular}{lccc}
\hline Tempo $(\mathrm{h})$ & $\mathrm{GM}$ & $\mathrm{GMT}$ & $\mathrm{P}$-valor** \\
\hline Basal & $85,71 \pm 8,40$ & $84,71 \pm 8,67$ & $\mathrm{P}=0,56$ \\
0 & $102,86 \pm 17,88$ & $101,86 \pm 13,48$ & $\mathrm{P}=0,56$ \\
1 & $99,57 \pm 19,54$ & $99,14 \pm 28,03$ & $\mathrm{P}=0,70$ \\
6 & $90,86 \pm 12,56$ & $99,43 \pm 13,23$ & $\mathrm{P}=0,22$ \\
12 & $89,71 \pm 14,12$ & $95,67 \pm 13,76$ & $\mathrm{P}=0,47$ \\
24 & $84,86 \pm 13,45$ & $91,33 \pm 6,50$ & $\mathrm{P}=0,52$ \\
P-valor* & $\mathrm{P}=0,18$ & $\mathrm{P}=0,28$ & \\
\hline
\end{tabular}

*Teste de Kruskal-Wallis ao nível de 5\% de significância.

**Teste de Mann-Whitney ao nível de 5\% de significância.

\section{CONCLUSÃO}

Com os resultados deste estudo, foi possível concluir que a utilização de meloxicam associado ou não ao tramadol, nas doses e posologias propostas, é apropriada no controle da dor pós- operatória de cadelas submetidas à $\mathrm{OVH}$ videoassistida com dois portais. Contudo, a resposta individual ao estímulo álgico pode tornar necessário resgate analgésico em determinados animais. 


\section{REFERÊNCIAS}

ALMEIDA, M.R.; LUNA, S.P.L.; ALVES, R.M. et al. Sobre a analgesia pós operatória da morfina, cetamina ou da associação em cadelas submetidas à ovariossalpingohisterectomia eletiva. Ciênc. Rural, v.43, p.1271-1276, 2013.

BASSO, P.C.; RAISER, A.G.; BRUN, M.V. et al. Ovariossalpingo-histerectomia em cadelas: comparação da dor e análises cardiorrespiratória, pressórica e hemogasométrica nas abordagens convencional, por NOTES híbrida e NOTES total. Arq. Bras. Med. Vet. Zootec., v.66, p.1329-38, 2014.

BONICA J.J. Pain research and therapy: history, current status and future goals. In: SHORT, C.E.; POZNACK, A.V. (Eds). Animal pain. New York: Churchill Livingstone, 1992. p.1-29.

BREAZILE, J. Physiologic basis and consequences of distress in animals. J. Am. Vet. Med. Assoc., v.191, p.1212-15, 1987.

COSTA, P.R.S.; ARAÚJO, R.B.; COSTA, M.C.; MAIA, R.E.N. Endoscopia gastroduodenal após administração de nimesulida, monofenilbutazona e meloxicam em cães. Arq. Bras. Med. Vet. Zootec., v.59, p.903-909, 2007

DAY, T.K.; PEPPER, W.T.; TOBIAS, T.A. et al. Comparison of intra-articular and epidural morphine for analgesia following stifle arthrotomy in dogs. Vet. Surg., v.24, p.522-30, 1995

FELDMAN, E.C. Hyperadrenocorticism (cushing's syndrome). In: FELDMAN, E.C. (Ed). Canine and feline endocrynology and reproduction. 2.ed. Philadelphia: Saunders, 1996. p.187-265.

FIRTH, A.M.; HALDANE, S.L. Development of a scale to evaluate postoperative pain in dogs. J. Am. Vet. Med. Assoc., v.214, p.651-659, 1999.

FREEMAN, L.J.; CHIOREAN, M.V.; CONSTABLE, P.D. Comparison of pain and postoperative stress in dogs undergoing natural orifice transluminal endoscopic surgery, laparoscopic, and open oophorectomy. Gastrointest. Endosc., v.72, p.373-380, 2010.

HANSEN, B. Torough a glass darkly: using behavior to assess pain. Semin. Vet. Med. Surg. Small Anim., v.12, p.61-74, 1997.

LAMONT, L.A.; TRANQUILLI, W. J. Physiology of pain. Vet. Clin. N. Am. Small Anim. Pract., v.30, p.703-728, 2000
LESTER, P.; GAYNOR, J.S. Management of cancer pain. Vet. Clin. North. Am. Small Anim. Pract., v.30, p.951-966, 2000.

LUZ, M.J.; BUSTAMANTE, S.B.; FERREIRA, G.S. et al. Dor em videocirurgia. Medvep, v.7, p.536-540, 2009 .

MATHEWS, K.A. Nonsteroidal anti-inflammatory analgesics in pain management in dogs and cats. Can. Vet. J., v.37, p.539-43, 1996.

McCORMACK, K. Non-steroidal antiinflamatory drugs and spinal nociceptive processing. Pain., v.59, p.09, 1994.

OTERO, P.E. (Ed). Dor: avaliação e tratamento em pequenos animais. São Paulo: Interbook, 2005. 293p.

PENNING, J.P. Pre-emptive analgesia: what does it mean to the clinical anaesthetist? Can. J. Anesth., v.43, p. $97-101,1996$.

PEREIRA, D.A.; MARQUES, J.A. Uso de morfina, xilazina e meloxicam para o controle da dor pósoperatória em cadelas submetidas à ovariossalpingohisterectomia. Arq. Bras. Med. Vet. Zootec., v.61, p.353-361, 2009.

RIBEIRO, S.; SCHMIDT, A.P.; SCHMIDT, S.R.G. O uso de opioides no tratamento da dor crônica não oncológica: o papel da metadona. Rev. Bras. Anest., v.52, p.644-651, 2002.

SHIH, A.C.; ROBERTSON, S.; ISAZA, N. et al. Comparison between analgesic effects of buprenorphine, carprofen, and buprenorphine with carprofen for canine ovariohysterectomy. Vet. Anaesth. Analg., v.35, p.69-79, 2008.

SMITH, J.D.; ALLEN, S.W.; QUANDT, J.E.; TACKETT, R.L. Indicators of postoperative pain in cats and correlation with clinical criteria. Am. J. Vet. Res., v.57, p.1674-78, 1996.

STEDILE, R.; BECK, C.A.C.; SCHIOCHET, F. Laparoscopic versus open splenectomy in dogs. Pesqui. Vet. Bras., v.29, p.653-660, 2009.

TEIXEIRA, R.C.R.; MONTEIRO, E.R.; CAMPAGNOL, D. et al. Effects of tramadol alone, in combination with meloxicam or dipyrone, on postoperative pain and the analgesic requirement in dogs undergoing unilateral mastectomy with or without ovariohysterectomy. Vet. Anaesth. and Analg., v.40, p.641-649, 2013. 